This item was submitted to Loughborough's Research Repository by the author.

Items in Figshare are protected by copyright, with all rights reserved, unless otherwise indicated.

\title{
Linguistic relativism: logic, grammar, and arithmetic in cultural comparison
}

PLEASE CITE THE PUBLISHED VERSION

http://dx.doi.org/10.1016/j.langcom.2006.05.001

\section{PUBLISHER}

(c) Elsevier

VERSION

AM (Accepted Manuscript)

LICENCE

CC BY-NC-ND 4.0

REPOSITORY RECORD

Greiffenhagen, Christian, and Wes Sharrock. 2019. "Linguistic Relativism: Logic, Grammar, and Arithmetic in Cultural Comparison”. figshare. https://hdl.handle.net/2134/14203. 
This item was submitted to Loughborough's Institutional Repository (https://dspace.lboro.ac.uk/) by the author and is made available under the following Creative Commons Licence conditions.

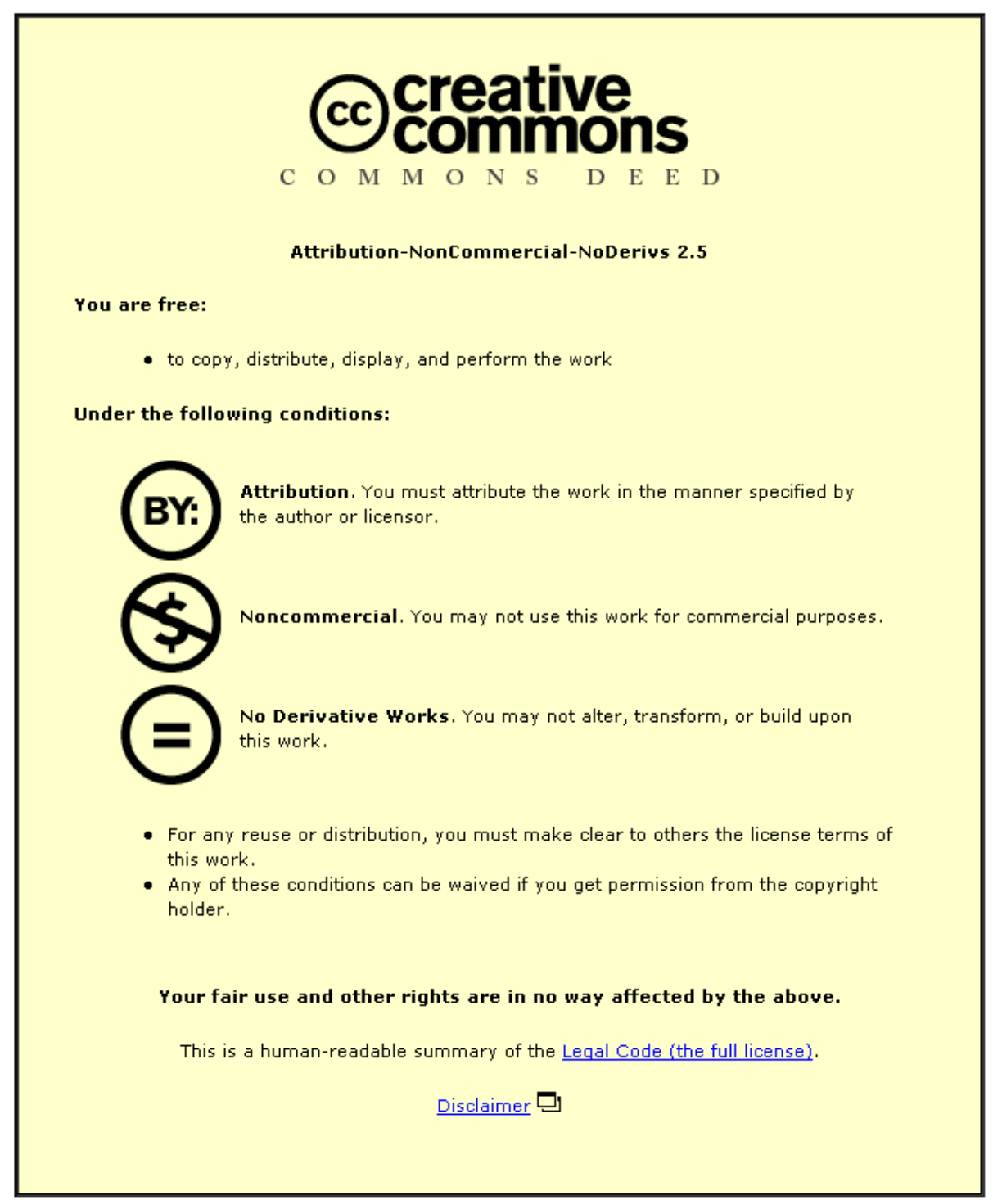

For the full text of this licence, please go to: http://creativecommons.org/licenses/by-nc-nd/2.5/ 


\title{
Linguistic relativism:
}

\section{logic, grammar, and arithmetic in cultural comparison}

Authors: Christian Greiffenhagen and Wes Sharrock

\author{
Address: \\ Christian Greiffenhagen \\ Department of Sociology \\ Roscoe Building \\ University of Manchester \\ Manchester M13 9PL \\ UK
}

Tel: +44 1612752497

Fax: +44 1612752514

Email: Christian.Greiffenhagen[replace_with_@]manchester.ac.uk

Version: May 11, 2006

Greiffenhagen, C. and W. Sharrock (2007). Linguistic relativism: logic, grammar, and arithmetic in cultural comparison. Language \& Communication 27 (1), 81-107. 


\title{
Linguistic relativism:
}

\section{logic, grammar, and arithmetic in cultural comparison}

\begin{abstract}
Linguistic relativism is the thesis that the grammatical structures of different languages imply different conceptions of reality. In this paper we critically discuss one form of linguistic relativism, which argues that grammatical differences between the English and Yoruba language exhibit differences in how English and Yoruba speakers 'see' reality (namely in terms of ‘spatiotemporal particulars’ and 'sortal particulars’ respectively).
\end{abstract}

We challenge the idea that linguistic relativism is an empirical thesis, i.e., a thesis that is substantiated through anthropological examples. We show that linguistic relativism is based on two assumptions: firstly, that the purpose of language is to describe the world; secondly, that being able to speak presupposes an ontological theory of the ultimate constituents of the world. We argue that the attempt to extract the outline of that theory from the language inevitably distorts the portrayal of language-using practice itself.

Greiffenhagen, C. and W. Sharrock (2007). Linguistic relativism: logic, grammar, and arithmetic in cultural comparison. Language \& Communication 27 (1), 81-107. 


\section{Introduction}

This article contributes to a critical analysis of some empirical examples that are given in support of cultural relativism, which we take to be the thesis that logic, grammar, and arithmetic are, in some sense, relative or only locally valid. Our interest lies in showing how hard it is to pin down just what 'in some sense' amounts to. In this paper we will focus on language and linguistic relativism ${ }^{1}$. As will be seen, however, the discussion modulates into a discussion of arithmetic, since the arguments about the relativity of language are used to motivate a case about the relativity of number.

The kind of linguistic relativism considered here involves the assumption that different grammatical structures and vocabularies imply different conceptual schemes or metaphysics, which postulate the ultimate constituents of the material world. For example, according to Whorf:

[...] the Hopi language and culture conceals a metaphysics, such as our so called naive view of space and time does, or as the relativity theory does; yet it is a different metaphysics from either (Whorf, 1956, p.58).

In other words, linguistic relativism is less interested in language per se than in investigating which ultimate constituents of the world are identified 'in' the language. The relativist's engagement with language is metaphysically motivated, based on the Whorfian assumption that each language accommodates a distinctive, unified metaphysics. This licenses the supposedly startling conclusion that inhabitants of different language communities inhabit worlds consisting of different kinds of things:

\footnotetext{
${ }^{1}$ In companion papers we discuss mathematical relativism (Greiffenhagen and Sharrock, 2006) and logical relativism (Greiffenhagen and Sharrock, forthcoming).
}

Greiffenhagen, C. and W. Sharrock (2007). Linguistic relativism: logic, grammar, and arithmetic in cultural comparison. Language \& Communication 27 (1), 81-107. 
When [Whorf] uses [...] phrases [such as] 'dissect nature' and 'the categories and types that we isolate from the world of phenomena', he is not thinking of fish and fowl or flora and fauna; he is thinking, rather, of the metaphysical categories: thing, event, relation, substance, quality, action, past, present, future, and so on. These are the categories into which languages 'dissect nature', and Whorf's relativism is the claim that languages differ in the way in which they deploy such categories. (Cook, 1978b, p.5)

We take it as plain that there are differences between languages, e.g., in their grammatical structures or vocabularies. However, we question whether this necessarily implies variable metaphysical commitments on the part of speakers of different languages. The reason for this is not because we think that the metaphysics are uniform across languages, but because we wonder (following Wittgenstein and Cook) whether speaking a language depends on a metaphysics in the first place. In other words, the question for us is not whether there are differences between languages (of course there are), but what this signifies, especially whether epistemological or ontological implications comparisons can be made of the basis of them - without, that is, the importation of questionable (at least contestable) philosophical assumptions to license reading these metaphysical implications into the materials ${ }^{2}$. Thus we agree with Cook who notes:

It is, of course, an empirical fact that spoken languages do differ greatly in grammatical structure, but it is an a priori philosophical claim that one can read off a metaphysics from grammatical structure. (Cook, 1978a, p.13)

In our view, the project of linguistic relativism only works by making several (unwarranted) assumptions about language. Firstly, it assumes that the (only) function or

\footnotetext{
${ }^{2}$ As one of us once put it: relativists "are the kind of people who would attribute a geocentric theory of the universe to us on the strength of the remark that we intend to get up tomorrow morning to watch the sun rise” (Sharrock and Anderson, 1982, p. 111).
}

Greiffenhagen, C. and W. Sharrock (2007). Linguistic relativism: logic, grammar, and arithmetic in cultural comparison. Language \& Communication 27 (1), 81-107. 
purpose of language is description. In other words, it assumes that language is a tool for naming the things in the world. This assumption has an important corollary: if the purpose of every language is description, then it is possible to compare whole languages of different cultures (as they share the same 'purpose' of naming things in the world).

Secondly, in a typical intellectualist manner, linguistic relativism stipulates that all description presupposes an ontological theory or a metaphysics - what Whorf (1956, p.221) calls an "unformulated and naive world view" and what Quine (1960, p.22) calls "the archaic and unconscious hypothesis of ordinary physical objects". In the form that we encounter it here this results in the primitive idea that the relationship between language and reality is effected by naming, and that the metaphysics of a language is manifested in the kinds of things that its names stand for.

In contrast, we see language as inevitably tied to a people’s culture, i.e., to the world that people live in and the practices that they engage in. Consequently, it is not possible to compare languages per se but only aspects of languages. When looking at the empirical examples offered by relativism, we find that the linguist either compares things that are ostensibly not the same (e.g., science and witchcraft) ${ }^{3}$ or that the compared things do not seem to be significantly different after all (without stipulating a metaphysics).

\footnotetext{
${ }^{3}$ This was already observed by Graves (1960) in his critique of an early form of linguistic relativism expressed by Lee (1960): "When discussing differences between the sound-complexes applied in various countries to the same reality, one should first make sure that it is the same reality. The words Brot, pain, and Pan are given only one meaning in an English polyglot dictionary: namely, 'bread'. But heavy German Brot, and light French pain, and hard Spanish pan are not all the same reality.” (p.155)
}

Greiffenhagen, C. and W. Sharrock (2007). Linguistic relativism: logic, grammar, and arithmetic in cultural comparison. Language \& Communication 27 (1), 81-107. 
In this article, we will try to exhibit that linguistic relativism is not an empirical but a philosophical project - and that it is possible to give an account of the examples offered by relativists without making the assumption that the relativist makes. We will focus on one central case of linguistic-cum-mathematical relativism, namely Helen Watson's ${ }^{4}$ initial attempts (e.g., Watson, 1987, 1990; Verran, 200a,b) to contrast Yoruba and English numbering. However, Watson is just one of many examples in the social studies of science literature that are based on the idea that language is description and that language embodies a theory of some kind (e.g., Bloor, 1976) ${ }^{5}$.

\section{Simplifying in order to create stark binaries}

Watson aims to exposit the different kinds of things encoded in the grammar of English and Yoruba. Her starting point is the assertion that

[...] predication leads language users to refer in particular ways and thus determines what kinds of objects their language defines as constituting the universe. I show that English and Yoruba (a West African language) predicate in different ways, and consequently postulate different kinds of objects. (Watson, 1990, p. 283)

Note that Watson is talking here about the whole of a language and not some specific aspects, e.g., names of snow, cooking utensils, or wedding ceremonies. Furthermore, Watson is comparing exactly two languages, English and Yoruba (rather than eight

\footnotetext{
${ }^{4}$ Helen Watson is now Helen Verran. We will throughout refer to her as 'Watson', citing, where necessary, her later papers as 'Verran'.

${ }^{5}$ It is also reflected in the deep rooted and general sociological suspicion of 'ordinary language' and in much of the long tradition of ideology critique. Many of the tendencies are crystallised in contemporary 'critical discourse analysis'. In these approaches 'ordinary language' is seen as embodying a theory, but an at best naïve, or, most often, a wrong one. The relativists share the assumption about the theory-dependence of language but are apt to think that all the theories are 'right'.
}

Greiffenhagen, C. and W. Sharrock (2007). Linguistic relativism: logic, grammar, and arithmetic in cultural comparison. Language \& Communication 27 (1), 81-107. 
languages, which might imply that she would have to specify how the languages are different and similar). Watson thus employs a typical relativistic strategy which we might call 'simplifying in order to create stark binaries'. Through creating gross synoptic characterisations of aspects of exactly two different languages, it is possible for Watson to make a simple and stark contrast. For example, she claims that:

Talking about the world necessarily involves postulating the type of constituents of the material world. (p. 288)

This stipulation is not taken as a starting point for a methodic exploration of how many different 'types of constituents' each language (English and Yoruba) postulates (perhaps three, five, or eighty-seven). No, it is further stipulated that each language will (with respect to numbers) postulate exactly one type of constituent. In other words, by limiting the possible choice of types of things to exactly one per language, Watson can reduce variations and align them along a single axis. This allows her to portray the two languages as standing in a simple contrast with respect to the types of things they postulate:

The difference in meaning [...] is a difference in the type of material object which is being postulated through use of a referring category. (p. 289; emphases added)

By reducing the differences between languages to one variable, all other differences are obscured. It is this simplification that enables the transition from a very few examined instances to an account of reference for a whole language that leads relativists as Watson to think that encountered differences have to be consistently contrasting ontological ones. If the analyst took greater care to look at the similarities and differences between 
languages, as well as the different kinds of differences within and between languages, the relativistic project would be much harder to get started.

\section{The ontological theory 'encoded' in language}

Linguistic relativism proceeds by stipulating a separation between words and concepts associated with words. The latter is supposed to refer to our ideas or theories about what makes the thing designated by the word what it is. For example, Watson (1990, p. 283) asserts that "how we count depends on how we conceive objects to exist". In other words, she assumes that our capacity to learn and use language requires possession of an additional theory of the world, namely a theory that specifies what it kinds of things our words - and numbers - refer to.

How can the analyst identify this underlying theory? For the relativist the answer lies in grammar. It is a further fundamental assumption of linguistic relativism that the grammar of a language reflects the form of the underlying concepts of a language. Hence a characterisation of the grammatical forms of a language should allow the anthropologist to make inferences about the concepts that language-users have of their world:

Whorf, like many philosophers, has the idea that one can read off a metaphysics from the grammar of a language and thereby discern something about the thinking of those who speak the language. (Cook, 1978b, p.7)

Whorf thinks of a metaphysics as something that is contained in a language as an inevitable feature of its grammatical structure. [...] he believes that grammatical categories lend themselves to a semantic interpretation employing metaphysical terms, such as thing, event, process, relation, past, present, future, and so on, so that to speak in a certain language just is to operate with, to think with, certain metaphysical categories. (Cook, 1978a, p.12)

Greiffenhagen, C. and W. Sharrock (2007). Linguistic relativism: logic, grammar, and arithmetic in cultural comparison. Language \& Communication 27 (1), 81-107. 
Just as we are not denying that there are differences between languages, so we are not denying that people have concepts and that these concepts may differ between different cultures (and differ in different ways). However, following Cook's (1978a,b) critique of Whorfian relativism, we challenge the idea of language as a conceptual scheme, i.e., the view that language embodies theories of reality. We are not denying that we have concepts, but we are claiming that these concepts arise within language and do not underpin it ${ }^{6}$.

For the relativist, the Whorfian assumption that the grammar of a language embodies metaphysics is the bridge from language to language users. In other words, it is the link from linguistics to psychology, sociology, and philosophy - from the structure of language to the ways in which people think (especially the way they think of reality). Anthropologists such as Watson are not interested in only exhibiting certain grammatical differences between languages (say, between the form and placement of otherwise equivalent number-words in sentences), but want to demonstrate that what these numbers mean (i.e., what species of material entity they are referring to) for language users is different.

\footnotetext{
${ }^{6}$ Neither are we denying that words have origins (etymology). However, we are questioning the role of that origin in current language-use. As Lenneberg (1953, pp. 465-466) observes: "There is a metaphorical element in language per se. The literal meaning of many metaphors, especially the most frequent ones, never penetrates consciousness, e.g., everybody, in the face of, beforehand, breakfast, inside, already. The translation method, however, distorts the significance of such forms of speech and often induces investigators to draw rather ludicrous conclusions." In short, the meaning of a word is fixed by its current use, not its origin.
}

Greiffenhagen, C. and W. Sharrock (2007). Linguistic relativism: logic, grammar, and arithmetic in cultural comparison. Language \& Communication 27 (1), 81-107. 
For the relativist, in order to be able to speak 'about' the world one has to be able to classify over the things 'in' the world. This in turn requires the possession of a theory 'of' the world (which specifies the criteria for the demarcation of the categories):

Learning to mean with language starts with classifying, but classifying does not amount to making meaningful sentences. Meaningful sentences are uttered within the framework of a particular theory of the world, a theory which the language used in making meaning necessarily and inevitably encodes. (Watson, 1990, p. 287)

In other words, for Watson it is necessary to have a theory of the world as a precondition of being able to acquire the language. This theory is encoded in the (grammar of the) language. Furthermore, this theory has the form of a proto-language:

Predication develops from the more complex of the classifying activities in what we might call 'proto-language use' - classifying over actions of bodies. (p. 287)

Thus, for the relativist, in learning language the child is moving from learning to classify things to learning to make meaningful statements. The child therefore must have made

a leap from proto-language to language; from making classifications to making meaningful sentences. A theory of the world has necessarily been engaged through adopting a particular way of classifying over actions of bodies in coming to use verbs, and the sorts of things that constitute the world are inevitably postulated. A specific sort of referring or designating category has been adopted. This particular type of referring category becomes an obligatory assumption of all those who use the language. (p. 287)

According to this picture, someone using language to talk about the world has to (implicitly) accept the classificatory assumptions encoded in the language ${ }^{7}$. In turn, the

${ }^{7}$ Of course this account begs the question of how someone is able to acquire the classifications without being able to speak. In other words: by explaining the acquisition of language through the possession of a 
analyst can work in the opposite direction, i.e., by investigating grammar the analyst can identify the theory embodied in the language:

We can identify and articulate what sort of a category it is by working from our understanding of the rules we use in using verbs - the criteria of our classification over actions of bodies, from which verbs grew. (p. 287)

In sum, this kind of linguistic relativism concerns divergences between languages that are not the usual readily recognised cultural differences, but instead are based on (radically) discrepant worldviews that are 'encoded' in the respective language. Schematically the logic of linguistic relativism can thus be summarised as:

- a theory of the constituents of the world underlies language;

- this theory is reflected in grammar;

- different languages have different grammars;

- therefore different peoples have different worldviews (where these worldviews are not expressed by what individual speakers say, but are built into the structure of the language in which they say whatever they say).

\section{Talking 'about' and 'in' the world}

Watson's unquestioning adoption of Whorfian assumptions seems to be reflected in her view of the role or status of language, namely that the fundamental function of language 
is description. In other words, language is seen as allowing people to talk 'about' the world. This is expressed in the title of a section of Watson's article:

\section{Talking About the World in English and in Yoruba}

In this section of the paper, I compare the types of material objects postulated as constituents of the material world by English-speakers and Yoruba-speakers as they engage in ordinary talk of the world. (Watson, 1990, p.288)

By conceiving of the purpose of language as descriptive, Watson is following Whorf, who argues that:

the Hopi language is capable of accounting for and describing correctly, in a pragmatic or operational sense, all observable phenomena of the universe (Whorf, 1956, p.58; our emphases).

In contrast, we would argue that ordinary people very rarely talk 'about' the world (but sociologists and philosophers often do). The affairs of people acting within practices would be better characterised as talking 'in' the world. It is an intellectualist fallacy to suppose that language was 'invented' in order to talk about the world - rather than as part of various cultural practices.

Watson seems to be an example of Quine’s (1960, pp. 28-30) ideal linguist in a foreign country, who is modelled after an (ideal type) natural scientist, i.e., as a disengaged observer. According to Quine's picture, this linguist adopts an objective or birds-eye perspective of the unknown culture and is then faced with the 'problem' of inferring from people's verbal behaviour what their sounds mean. Note that in this picture, there is no place for what people actually do, e.g., whether they engage in practices that familiar or unfamiliar to the observer. No one is eating, drawing, or raising rabbits - all they do is talk 'about' the world. 
Watson seems to suggest that people are already thinking about the world even prior to expressing their thoughts in language. It seems that we discretely first learn words and then learn how to apply them:

An English speaking child learns to chant 'one, two, three...', and a Yoruba speaking child learns 'eni, eji, eta'. Gradually the songs of number names become meaningful tools for manipulating the material world. What are the meanings that these words take on as they cease to be a mere chant? (Watson, 1987, p.339)

Number, according to Wittgenstein comes not from the world, but from our talk of the world. (Watson, 1987, p.351) ${ }^{8}$

In other words, this picture of language is associated with a peculiar picture of language learning. It is as though when being taught the words children had to perform an additional (cognitive) task which is to figure out what sorts of things those words could be talking about. According to this picture, learning the words comes first, followed by learning to apply them. However, this picture of language, involving an understanding of meaning as a matter of associating words with the things they stand for, is exactly the picture of language that Wittgenstein (often cited by relativists) argued against. It suggests that there is a level of thoughts (or meanings), either on the individual or the society level, prior to language. Language, on this account, is a way of expressing these thoughts (or to communicate them).

In contrast, we would argue that concepts are acquired from language. In other words, thoughts and language co-evolve (without being able to talk it makes no sense to speak of

\footnotetext{
${ }^{8}$ Bloor (1976, p.139) expresses a similar view: “A young child is taught the word 'hat' and has learned to recognize some hats.”
}

Greiffenhagen, C. and W. Sharrock (2007). Linguistic relativism: logic, grammar, and arithmetic in cultural comparison. Language \& Communication 27 (1), 81-107. 
thoughts). Furthermore, language is tied to a culture's activities. Language co-evolves with activities and it is only in those activities that language-use possesses its sense. Language is not a way to talk 'about' the world, but to carry out activities. In other words, the 'purpose' of language is not description but is tied to other cultural activities. Consequently, naming things in the world is not the purpose of language but only, so to speak, a preparation:

One thinks that learning language consists in giving names to objects. Viz, to human beings, to shapes, to colours, to pains, to moods, to numbers, etc. . To repeat - naming is something like attaching a label to a thing. One can say that this is preparatory to the use of a word. But what is it a preparation for? (Wittgenstein, 1953, §26)

Naming is so far not a move in the language-game - any more than putting a piece in its place on the board is a move in chess. We may say: nothing has so far been done, when a thing has been named. (§49)

In sum, speaking is tied to doing. In Wittgenstein's words: “the speaking of language is part of an activity, or of a form of life” (§23).

As outlined above, by stipulating that the purpose of language is description, the relativist is enabled to compare languages wholesale. However, if we see language not as independent of a culture and of the assorted practices it accommodates, then it is more difficult to compare languages without reference to one or other of the 'language games' that are played - and the differences that do exist may turn out to require complex, not sweeping, comparisons.

\section{From numbers to number names}

The assumption that the only purpose of language is description can be illustrated by anthropological studies of numbers, which focus not on how numbers are used as part of 
various practices (e.g., selling goods) and whether this use is different in the respective cultures, but that instead investigate how number names are linguistically formed. In this context, number names are the words that are used to pronounce the numbers when these are spoken - e.g. in English, 'one’, 'two', ‘three’, and so on.

Whilst relativists claim that what numbers 'are' may be different in different cultures, they typically do not claim that there are any significant computational incompatibilities in the practical affairs of everyday lives (e.g., within the trade between two different cultures). For relativists such as Watson (but also, Reed and Lave, 1979), although the applications of numbers in respect of, inter alia, measuring quantities and determining price are equivalent, there is nonetheless a supposedly significant difference between what, for example, English and Yoruba numbers refer to. As these differences will not show up in the practical affairs of everyday life, the only place where they are visible is in the structure of the language, in particular its grammar:

The number system can thus be shown to be intimately related to the conceptual frame that the linguistic structure lays down. (Watson, 1990, p.283)

The relativist moves from numbers as part of various practices of computation to number names as grammatical forms (since these possess linguistic structure and thus can be contrasted). In other words, accepting Whorfist suppositions about the relation of grammar to ontological-intellectualist worldviews on the part of actors, the relativist is able to make a subtle but vital shift from talking about 'numbers' to talking about 'number names'. Thus the question "how Yoruba and English numbers are different" (Verran, 2000b, p.346) is supposed to be answered by talking about "number names” (p. 346) and "number name formation" (p.350). This is done by 'translating' from the comparison. Language \& Communication 27 (1), 81-107. 
indigenous language not into English, but into the (neutral?) Arabic numeric system (on the right hand side in the following two tables):

$\begin{array}{ll}\text { Vai } & \text { Gloss } \\ \text { lundo } & 1 \\ \text { soolu } & 5 \\ \text { soolundo } & 5+1 \\ \text { tan } & 10 \\ \text { tansolundo } & 10+5+1 \\ \text { (excerpt from Reed and Lave, } 1977, \text { Table 2, p.573) }\end{array}$

$\begin{array}{lll}1 & \text { kan } & 1 \\ 2 & \text { méji } & 2 \\ 10 & \text { méwa } & 10 \\ 11 & \text { mókònlaa } & (+1+10) \\ 15 & \text { mééédoguin } & (-5+20) \\ 20 & \text { ogun } & (20)\end{array}$

(excerpt from Verran, 2000b, Table 1, p.347)

The move from numbers to number names in both Reed and Lave (1979) and Watson enables the Whorfian assumption that language embodies a theory of the constituents of the world to enter into the portrayal of the examples. The difference is not to be sought in the use of numbers in everyday activities, but in the associated concepts on the parts of language users. The concentration seems to be on what speakers mean, treated as something independent of how they act. In other words, the move from numbers to number names exhibits the Whorfian assumption that language is description:

A category such as number (singular vs. plural) is an attempted interpretation of a whole large order of experience, virtually of the world or of nature; it attempts to say how experience is to be segmented, what experience is to be called 'one' and what 'several'. (Whorf, 1956, p.137) 
Number names seem to be treated by relativists as if their linguistic form displays cognitive processes involved in their use. It is suggested that when the Yoruba say “fifteen” they think 'take five from twenty', whilst we think 'ten plus five' or 'add five to ten’. It is as if an English speaker saying 'eleven’ is really saying 'ten plus one' and must (mentally) make that computation whenever he or she says 'eleven'. This raises the root question: How far can one treat formal structural properties of a language system as entering into the sense of what its users say and mean?

The relativist assumes, firstly, that being able to count presupposes a metaphysical conception of the constituents of nature (being able to identify the items that are to be enumerated), and, secondly, that these concepts are captured in the grammar of number names (i.e., in their linguistic form). Again, it is simply stipulated that these translations capture how the people in a culture calculate. For example, Reed and Lave (1979, p.574) write:

when we add 17 and 3 , we have a single 'carry the 1' to do; Vai and Gola have two analogous steps to perform: $(10+5+2)+(3)=10+5+$ $5=10+10=20$.

But surely people competent in basic arithmetic (in either language/culture) can add 17 to 3 without any operations (seventeen and three is twenty). However, within the relativist's framework, any application of number is organised through some mental-conceptual operations on the part of actors. Again: How do relativists know which mental operations are being performed - except by hypothesising them from, and reading them back into, the differences of linguistic form? 
Let us again look at an example closer to home, e.g., English and French numbers (or number names). In French, 80 is 'quatre-vingts’ (“4x20”) and 90 'quatre-vingt-dix’ (“4x20+10”). Following the relativists’ argument, we must conjure cognitive operations out of the forms of these number words. In that case, when the French say 'quatre-vingtdix' they must accompany this by mentally adding ten to four times twenty. Again, that different languages have different names for their numbers comes as no great surprise. However, do these different names imply that different cultures calculate differently or that their practical calculations are accompanied by a necessarily distinctive sequence of computations? Do the French have distinctive computations or more problems in adding $82+82$ than the English? Do they even go through different computations than the English?

\section{From number names to theories of number}

As we have already shown with the example of the transition from numbers to number names, it is crucial to Watson's argument to fuse number systems with the ontology embodied in the language. Watson makes another attempt to connect Yoruba ontology into its numbering system by drawing on (meta-) mathematical formalisations of number systems. Watson is interested in these metamathematical formalisations of number systems, since one might be more akin to the English or Yoruba ontology encoded in language. Her treatment of this issue reinforces our point about the descriptive limbo in which her renderings exist, neither describing the operational procedures of a grammatical or mathematical system nor the actual practice of computations.

Greiffenhagen, C. and W. Sharrock (2007). Linguistic relativism: logic, grammar, and arithmetic in cultural comparison. Language \& Communication 27 (1), 81-107. 
Watson compares von Neumann's set-theoretic formalisation of the number system with Zermelo's, and announces that

As an English-language user of number, von Neuman's [sic] account seems to me to be intuitively correct. [...] In contrast, I suggest that a Yoruba speaker would choose Zermelo's account of number as correct. (Watson, 1990, p. 307)

Note again the strategy of 'simplifying in order to create stark binaries': on Watson's account there are exactly two possible ways to formalise numbers. This allows Watson to 'discover' that one fits the English and the other the Yoruba conception of number. However, and more importantly: Are we here dealing with a preference that any actual Yoruba speaker has ever had? Or are we dealing with a preference that Watson 'reads into’ ordinary practices (cf., Macbeth, 2002)?

The claim that any other English speaker would concur with her intuition and prefer von Neumann's account is easily disputed, since many English speakers would see no prima facie reason to prefer either one or the other formation as 'intuitively correct'. In our view, no English speaker need think that either account "would 'jump out' as the intuitively correct account” (p.308) - at least not without prior briefing in what is involved in philosophical-mathematical arguments. We would argue, rather, that neither von Neumann's nor Zermelo's account is distinctively 'correct', as both are 'correct' in terms of the standards that can be used to assess the adequacy of a formalisation as a representation of that which it formalises. Watson here engages in what we call the 
'ontologising of mundane phenomena'9 . Mathematically speaking, the number sequence which the von Neumann and Zermelo schemes respectively formalise is, of course, one and the same (it is the sequence of the natural numbers: $1,2,3$, and so on) ${ }^{10}$. Further, each of these two formalisations is capable of reproducing the form of succession of that sequence expressed in terms of relations between sets (but applying set theory in two different ways). There is therefore no mathematical reason why either of these should be judged to better present the number sequence to English speakers, nor to suppose that only one of them could be correct (why suppose that there must be only one way, or exactly two ways, in which to formalise the natural numbers?).

Thus, there can be no question as to whether either scheme could give the impression of representing the English number system in a better or worse way. Questions about which one is preferable are inevitably technical mathematical-philosophical ones, of which the average English or Yoruba speaker will be wholly unaware, and which could make no difference to their calculating practices were they to be advised of them. To see what the disagreement over the two formalisations might involve (which is at no point a

\footnotetext{
${ }^{9}$ In a later paper (Verran, 2000b, p.364) she seems to acknowledge this: "For years as I puzzled about the differences between Yoruba and English numbers, I remained convinced that I had accidentally stumbled across all the signs of the existence of the object - Yoruba numeration. I failed to notice that if I had just used numbers, say in buying provisions for my family in the market, concentrating only on the price of the bowl of rice (insisting loudly that the vendor heap it up) or pile of tomatoes (noting in my negotiations their over-ripeness), bargaining with the vendor to get a better price, there would have be no resources to make an object with. It is precisely that I am bringing the object of the Yoruba numeration system home to the academy rather than just the rice and tomatoes home to my family, which provides the materials to generate the object 'Yoruba numeration system'.”

${ }^{10}$ Even Quine recognises this: “One uses Frege’s version or von Neumann's or yet another, such as Zermelo's, opportunistically to suit the job at hand, if the job is one that calls for providing a version of number at all. [...] Each of these three progressions or any other will do the work of natural numbers, and each happens to be geared also further jobs to which the others are not.” (Quine, 1960, p.263)
}

Greiffenhagen, C. and W. Sharrock (2007). Linguistic relativism: logic, grammar, and arithmetic in cultural comparison. Language \& Communication 27 (1), 81-107. 
mathematical disagreement) one would need to acquaint oneself with philosophicalmathematical conceptions about what is at stake in the attempt to give satisfactory formalisation of the nature of number. In other words, one would engage with the philosophical-mathematical foundations of number and debates among, inter alia, Frege, Boole, Russell, Hilbert, Wittgenstein, Brouwer, and so on. However, the different philosophical positions do not align with grammatical, cultural, or psychological differentiation of English or Yoruba.

Watson (1990) says about English:

As an English-language user of number, von Neuman's [sic] account seems to me intuitively correct [about English number words]. Cardinality, on his account, seems to encode a one-by-one collection of predecessors [...] A number, in this version, is the last number of the series reached through one-by-one progression. (p. 307)

In contrast,

[...] a Yoruba speaker would choose Zermelo's account of number as correct. [...] In this version, each number is totally subsumed by its successor, and any one number has a unified nature. I contend that for a Yoruba speaker, the model of the number which would 'jump out' as the intuitively correct account would be this one, for Yoruba-language numbers carry 'the flavour' of a divided whole; there is no sense of a linear stretching towards the infinite here. (pp. 307-308)

Whilst these picturesque characterisations may have illustrated the sense in which Watson reads Zermelo's account of set-theory as corresponding to her picture of Yoruba and English ontologies, she does nothing to demonstrate that the construction, understanding, or use of that formalisation plays a role on the part of actors. In other words, we would like to see an explanation of how the imagined ontological content might be shown to be integral to the mathematical constructions themselves. We would 
like to see a demonstration of how it is that, in mathematically relevant ways, Zermelo's formalisation categorically lacks what von Neumann’s allegedly possesses, namely “a sense of a linear stretching towards the infinite”. We would also welcome a detailing of how "'the flavour' of a divided whole" is to be found in one but not in the other. We do not say that such an account could not be persuasively given, but that its persuasiveness would scarcely hinge upon the instantly intuitive transparency of the initial characterisation.

What is notably absent from Watson's (as well as Reed and Lave's) accounts of numbers is any use of numbers in daily life, i.e., a demonstration that in their daily lives the Yoruba count differently from us. The comments that the authors do make show consistently the contrary, namely that the mathematical operations remain the same. For example:

Among other things, Yoruba-speaking people buy and sell oranges, rope and beverages, plots of land and nuggets of gold. The actions they use in these exchange transactions are familiar and recognizable to English speakers who, by watching, can easily render in Englishlanguage terms what has been bought and sold [...]. (Watson, 1990, p.294; emphasis added)

And:

The English-speaking visitor to Yorubaland, and the Yoruba-speaking visitor to England, feel a comforting familiarity while watching the foreigners ascribing value during the exchange of goods. Their actions show that they are using the familiar ways of perceiving 'the amount of stuff here and now'. Although the talk of foreigners cannot be understood, and the ways percepts are talked of and the things they are said to be are unknown, a stranger may still successfully participate in the exchange. (Watson, 1990, p.292; emphasis added) 
In other words, the supposed differences in ontologies are not the kinds of differences that show up the practical affairs of everyday life. Despite the supposedly significant differences between English and Yoruba, it is possible to trade between the two countries without misunderstandings - and it is possible for an English person to visit the Yoruba and make sense of what they do (while allegedly not really understanding what they say).

\section{Different ontologies: spatiotemporal and sortal particulars}

After this illustration of Watson's rationale for moving from ordinary words and ordinary practices to ontological schemes (or Weltanschauungen) we will now try to outline what we understand the respective ontologies to be, since these are not spelled out in any coherent way (nor is there much attempt to show how this supposed difference cashes out).

Watson aims to surprise her readers by showing that the difference between these two languages is other than they had supposed it. Rather than showing that these two languages speak of the same things in different ways, Watson wants to show that these two languages speak of different things:

Analysing the workings of predicators in English-language usage and in Yoruba language-usage to infer the extension of designating terms used in utterances in those languages, leads me to a conclusion that some may find rather startling: namely, that speakers of these languages talk of different types of things when they designate over the material world. (p. 289; emphasis added)

Of course everything hinges on how 'speaking of things' is to be understood. We do not find it easy to fix on just how English and Yoruba are supposed to differ and our attempt 
to do this immediately follows accompanied by some comments on the difficulties we have.

Recall that Watson's eventual target is the referential status of numbers. Furthermore, recall that Watson does not aim to show that Yoruba and English mathematics are different in the sense that they generate different results (i.e., five plus seven gives twelve in English and Yoruba mathematics). Instead, Watson wants to prove that what the numbers 'are' (what they signify) in Yoruba is different from what they refer to when we use them. In other words, in both English and Yoruba $5-3=2$ but the kind of fundamental entity the '2' refers to differs in each case. Hence, Watson is not concerned with practical language use or application of mathematics in everyday computation. Instead, she postulates a difference in the way objects are classified (though that difference does not entail divergence in the criteria of individuation for everyday objects):

I have proposed that referring develops from the practice of juxtaposing two types of classifiers - classifiers over types of bodies, and classifiers of the types of action that bodies engage in. The type of classification wrought with respect to interaction between bodies will determine the type that the referring category is. In English we have verbs, which code the interaction between bodies on the basis of spatially individuated bodies taken to endure across time, as the interacting element. The referring category created by the functioning of verbs in the code which is the English language is the category of spatiotemporal particulars.

In the Yoruba language, interaction between bodies is coded as one type of matter interacting with other types of matter; the interacting element is treated as a particular sort of matter. This type of referring category implicit [emphasis added] in Yoruba-language use is the category of sortal particular (Watson, 1990, p. 289). 
We find it difficult to determine what Watson is claiming here: Is she remarking on the linguistic structure of these two languages? Is she making some (strange) metaphysical stipulations about language? Is she talking about aspects of the Yoruba language or culture that might be unfamiliar to the English? Firstly, note again the use of the strategy 'simplifying in order to create stark binaries', i.e., the availability of precisely two types of classifiers - which then allows Watson to map exactly one classifier onto each language (just imagine the complications for her argument if there were three, or even five, types of classifiers). Secondly, note the movement between grammar and ontology, i.e., between talking about verbs and talking about "spatially individuated bodies taken to endure across time”. It is on the latter level that Watson wants to discover differences:

Because of the different classifications which lie behind the functioning of 'is' and ' jé' as predicators, an English speaker ends up referring to a spatially separated and temporally located material object, and a Yoruba speaker ends up referring to a section of matter that is created around a certain set of characteristics - matter of a certain sort. (p. 289)

Watson's 'startling' conclusion: the English and Yoruba talk about different kinds of things. We would like to get a clearer idea of what the difference in kind is supposed to be. Here is Watson on English:

The material objects which English-speakers talk of are spatiotemporally defined objects of different sorts. For English speakers, the primary criterion by which the objects talked of are defined is the idea of matter set in relation to the idea of space-time. Secondarily, these objects are categorized along sortal lines. Features that spatiotemporal things are said to have name the different inherent characteristics which things of different sorts are supposed to have. English-speaking people come to say that the spatiotemporal particulars that they talk of have 'qualities'. Qualities do not purport to name material objects. Toes cannot be kicked against qualities, nor fingers dipped into them. 
The idea of amount of matter enters English-language talk through talk of qualities. The extent to which some qualities are held or exhibited by spatiotemporal particulars may be taken as varying concomitantly with the total amount of matter which constitutes that spatiotemporal particular. These qualities are those which are most commonly exhibited: numerosity, volume, area, length and weight. In ordinary English-language quantification, the extent to which any of these qualities is exhibited by a spatiotemporal particular, may be reported with a number. That number is taken as reporting 'the total amount of matter manifesting'. (p. 293)

To the best of our understanding, Watson is trying to exhibit the structure of the metaphysics embodied in the English language. According to her, the basic 'building blocks' are spatiotemporal particulars. These spatiotemporal particulars have qualities that are not themselves spatiotemporal particulars. English-speaking is apparently seen to involve referring to (so to speak) individually independent entities. These entities are supposed to be items that are discrete from all others and whose identity is exhausted by their existence at a particular time and a determinable place.

It seems that Watson is talking about a fairly straightforward atomistic ontology here: there are things in the world and these things possess definite qualities, such as length and weight. What is perhaps a bit surprising is the inclusion of numerosity among the qualities: are two pigs 'one' thing with the numerosity 'two' - or 'two' things each with the numerosity 'one'? Further, note the way that identification involves primary and secondary identifications. We wonder how such a treatment of English might apply to 'paper cup', 'rubber ball' or 'toughened glass'. Finally, it seems that a sophisticated theory of the world is needed in order to be able (say, for a child) to speak about pigs and horses.

In turn, the ontology embodied in the Yoruba language is described as follows:

Greiffenhagen, C. and W. Sharrock (2007). Linguistic relativism: logic, grammar, and arithmetic in cultural comparison. Language \& Communication 27 (1), 81-107. 
The object which Yoruba speakers are committed to saying there are in the world are sortal particulars - material objects defined through their particular nature. Certain sets of characteristics form definitive boundaries of the material objects that Yoruba speakers talk of; the objects talked of are construed as being infinitely scattered through space and time. The here/now-ness or then/there-ness of a manifestation is irrelevant in creating an object to talk of. We could say that qualities are the boundary lines of the objects primarily talked of by Yoruba speakers. Sortal particulars cannot be ascribed qualities, since it is on the basis of a qualitative distinction that they come into being as objects to talk of.

When one speaks of sortal particulars, and one wants to say more about them than merely that they manifest in their characteristic ways, it is on their mode of manifesting that one must comment. Mode is the manner in which a sortal particular manifests - a here/now or then/there (spatiotemporal) slice of a sortal particular. When sortal particulars are the primary material objects that one speaks of, the modes in which they may be said to manifest are features of these objects. In quantification in Yoruba-language talk, it is through mode that number comes to report the idea of total amount of matter present. Valuation over matter is a form of modification in Yoruba-language talk. (pp. 297-298)

Perhaps for Watson this means something like the following: the Yoruba do not have an atomistic ontology, but instead have a continuous or flowing one ${ }^{11}$. In other words, the stipulated 'building blocks' of the Yoruba ontology are not separate, discrete items - but are continuous bodies (blocks?) of 'matter'. For the Yoruba, the world supposedly consists not of individualised units of the sort that English-speakers identify as objects. Instead, the world for the Yoruba is said to consist fundamentally of what Englishspeakers might term substances. It is out of these substances that observable things (what,

\footnotetext{
${ }^{11}$ Watson seems to be following Quine's discussion of (theoretically) possible translations of the imagined word 'gavagai' (apart from simply as 'rabbit'): “A further alternative likewise compatible with the same old stimulus meaning is to take 'gavagai' as a singular term naming the fusion, in Goodman's sense, of all rabbits: that single though discontinuous portion of the spatiotemporal world that consists of rabbits. [...] And a still further alternative in the case of 'gavagai' is to take it as a singular term naming a recurring universal, rabbithood.” (Quine, 1960, pp.51-52)
} 
in English would be identified as objects) are made. Each fundamental substance appears to comprise one single extensive and unified entity, which is continuous and infinitely spread' through space. On this account, observable things are localised materialisations (pun intended!) of the continuity of these substances, and are not, therefore, free standing and discrete entities. Observable things then are part of the one (or more?) substance(s) of which they partake. Thus, they are not genuine units, but only - Watson's word ‘slices' or 'sections' of the basic substances (note the metaphoric character of 'slice' and 'section').

For Watson, the English-speaking idea of an object seems applicable in Yoruba - if at all - only to the fundamental substances (though something which is infinitely spread would not easily qualify as a 'bounded entity'), for observable things retain their connection with the substances of which they are part. Thus, what English would designate an object is only a localised fragment from the single underlying substance, and is recognised to be such by Yoruba-speakers. For Watson, when the Yoruba (seemingly) speak of any individual thing, they actually speak of it as a subdivided extract from the unified substance. This explains Watson's rather strange translations, of, e.g., "It is water” as

"Watermatter (matter with the characteristics of waterness) here manifests its inner, intrinsic and permanent nature” (p.289).

Or "He gave me four stones" as

"He gave me matter with characteristics of stoneness manifesting here/now as a collection divided to the extent of four" (p. 298). 
Note again the very elaborate ontology necessary on part of language-users - but also the possibly problematic relation between what a speaker is saying and what, according to Watson, the speaker must be meaning (see below).

Having postulated two different ontologies, Watson now moves on to numbers:

Collections of individual things are said to have the quality of numerosity to a certain extent. Cumulations of continuous matter are said to have the qualities of length, area, volume or weight to varying extents. When numerosity is used as the basis of quantification, the process is called 'counting'. (p. 294)

In English-language talk, number is a concept which organizes over the concept of qualities, which in turn organizes over the concept of spatiotemporal particulars. (p. 300)

In other words: spatiotemporal particulars are supposed to have qualities (e.g., numerosity or length) which can be measured. Thus, numbering in English is (for Watson) a matter of serially attaching equally discrete numerals to a plurality of singular countable items. In contrast:

Yoruba speakers talk of a particular sort of continuous matter, an object spread infinitely over the here/now-then/there. In generalizing over this category, they come to talk of mode, a 'slice' of a sortal object, bounded by the here/now or then/there. [...] Number, in Yorubalanguage talk, is a degree of dividedness within here/now-then/there slices of sortal particulars. In Yoruba-language talk, number organizes over mode, which in turn organizes over the sortal particulars. (p. 301)

For Watson, numbers among the Yoruba-speakers number how many subdivisions have been made within a greater whole.

As far as we can understand what Watson is trying to say here, it seems to boil down to something like the following: within the English atomistic ontology the world already comes in objects which, inter alia, have the quality of 'unit'. Hence, counting means 
'collecting'. In contrast, the Yoruba continuous ontology does not have such discrete objects but continuous substances that turn up in bits. Thus counting for the Yoruba is ‘subdividing'.

\section{How is the comparison done?}

Watson's overall goal is to exhibit differences in the ontologies embodied in the respective languages. It seems to us that there are two possible ways of doing this. The first would involve a neutral or independent standard according to which aspects of both languages can be compared. The second would involve the direct translation from one language into the other (without making recourse to an intermediate, independent standard).

\section{(a) Neutral/independent standard}

The first is a familiar way of proceeding in fields such as phonetics or cognitive anthropology. This method was implicitly employed, firstly, in the discussion of different number names above (where the Arabic numerals constituted an independent standard of English and Yoruba number words), and, secondly, in the theories of numbers 'embodied' in the language (where set theory was used to characterise both von Neumann versus Zermelo formalisations of the natural numbers).

If there was an objective language available for describing worldviews or metaphysics, then that language could be used to compare instances of concrete realisations, e.g., between the English and Yoruba metaphysics. However, despite attempts by logical positivists to develop such a language, it is generally not in any way regarded as standard

Greiffenhagen, C. and W. Sharrock (2007). Linguistic relativism: logic, grammar, and arithmetic in cultural comparison. Language \& Communication 27 (1), 81-107. 
(and Watson does not seem to employ it in her argument). Furthermore, such a standard would itself not be relative, but absolute (and thereby refute the relativistic thesis).

Another possible way would be to see grammatical categories as constituting such a neutral standard, e.g., 'verb’ or 'adjective' as designating concepts that are languageindependent $^{12}$. In that case, Watson could proceed as follows: an ontology of the world is needed in order to be able to speak; this ontology is reflected in the grammar of a language; linguistics has provided us with a 'neutral observation language' to describe the grammar of a particular language; given this grammatical description we can then 'read off' the ontology from the grammar. Watson sometimes seems to be proceeding along such lines:

The grammatical mechanism through which the sortal particulars that Yoruba speakers talk of are modified in the operation of ascribing value involves the incorporation of nominalized verb phrases in an elided form. (p. 298)

However, for us it seems problematic to attempt to extrapolate from grammatical features of language to supposed ontological presuppositions of language. Why should familiar grammatical differences necessarily project 'different kinds of objects'? We have in mind, for plain example, the differences between English, French, and German and their

\footnotetext{
12 This again raises the problem that the categories of grammar would then seem to be absolute and not relative. As Joseph (2002, p.104) puts it: "It seems clear enough that Sapir and Whorf share with the analytic philosophers and semanticists a fundamental belief in a transcendent, universal logic which no 'natural' language captures perfectly. Whorf also holds at times that some languages capture some aspects of this logic better than others, as when with regard to tense in Hopi he does not describe it as constructing time in an alternative and equally valid to that of 'Standard Average European', but in a way that would make Hopi a superior vehicle for the formulation of quantum physics. Thus his 'linguistic relativity principle' fails to be relativistic in two senses, since it is grounded in a belief in 'true' logic, and does not decline to pass judgement on how well particular languages embody that logic.”
}

Greiffenhagen, C. and W. Sharrock (2007). Linguistic relativism: logic, grammar, and arithmetic in cultural comparison. Language \& Communication 27 (1), 81-107. 
number of articles/genders: one ("the") in English, two ("le, la") in French, and three (“der, die, das”) in German. For certain purposes (e.g., teaching a foreign language) it might make sense to translate the French "la pomme" into "she apple" and the German “der Apfel” into "he apple” to illustrate the different grammatical forms in the languages. However, why would we want to say that this has metaphysical implications? Should the latter be really be translated to "here is an apple in all its masculinity" in order to convey to English-speakers that in German, apples have male genitals, and French apples have female ones? Do English, French, or German people have different experiences when actually eating an apple? Do they think about a different kind of object when talking about an apple? In which sense could we say that 'she apple' or 'he apple' means or says anything in English that is not perfectly satisfactorily translated by 'apple'? In our view, neither 'he apple' nor 'she apple' are good renderings into English, since they do not mean anything in that language. What they mean in German and French is fully given by translation into English as ‘apple’.

In our view, it is the words that are gendered not necessarily the things that the words may be identifiers for. Knowing the gender of a word provides a rule for grammatical formation of constructions using those words (for example, pronouns used, endings attached, or matters of complementation). The same point applies to, for example, the active and the passive. It would be naïve to think that variations between active and passive constructions featuring a verb automatically indicates a difference with respect to either what was done or how it was done. Active and passive forms can often both and indifferently identify the same action.

Greiffenhagen, C. and W. Sharrock (2007). Linguistic relativism: logic, grammar, and arithmetic in cultural comparison. Language \& Communication 27 (1), 81-107. 
However, despite occasional references to the grammatical structures of the respective languages, Watson typically bypasses grammar and directly attempts to talk about the implied ontologies. Thus, in our view, Watson does not proceed in this first way of comparison. Instead, Watson's arguments are meant to take their strength from the way Yoruba expressions translate into English.

\section{(b) Translation from one language to another}

One might suppose that translation from Yoruba to English would be very difficult since according to Watson there is a significant difference in the respective ontologies. It is also worth recalling that Watson is not reporting some hitherto unknown tongue with no prior English translations, but comparing two languages where translation has been an ongoing affair. In fact, dictionaries and introductory textbooks are available (e.g., Barber, 1984).

Watson bids to secure the adequacy of the translations as the paper was reviewed by competent Yoruba speakers who complimented their quality:

Translations of Yoruba-language utterances into English are crucial to the argument in this paper. However, only the exceptional reader will be in a position to evaluate the translations. A reader might well question the veracity of the translations, and hence be quite unmoved by the argument. Here readers must rely on the referees of the paper who speak Yoruba. [...] Since the rendering of Yoruba language utterances into English is crucial, I make several attempts in English to catch the subtleties I am trying to focus upon. The first translation conveys most precisely what is encoded in the Yoruba utterance; subsequent glosses work towards less clumsy and more acceptable English. (Watson, 1990, pp. 310-311, endnote 15; our emphasis).

At the same time, Watson points out that her English translations differ from those which might be conventionally and readily given for Yoruba expressions. Her translations are 
meant to render "what is encoded in the Yoruba utterance" (p.311; our emphasis). She describes some of her English representations of Yoruba as "literal translations" (e.g., p. 289). However, it is not clear to us that this is what they are.

Watson, again, seems to following in the footsteps of Whorf and Sapir who used the method of 'conceptual translations':

Sapir, for instance, says that when a sentence from another language has been thus translated into distorted English, it has been 'conceptually translated'. By this he seems to mean that we can get at the meaning of the original sentence by means of the piece of distorted English. [...]

Why, then, does Sapir call it a translation, and why a conceptual translation? It is because he intends that we should read through 'It stones down' as though we were reading in some language, in order that the 'meanings' of the grammatical categories of Nootka shall be registered in our minds as they are in the minds of the speakers of Nootka when they use the (actual) Nootka sentence. This is why Sapir calls it a 'conceptual translation': he thinks that it reveals Nootka 'concepts' or 'meanings'. (Cook, 1978b, p.10)

Of course, the problem with such 'conceptual translations' is to specify the range of applicability: does it apply to every feature of grammar (including, for example, gendered nouns in German and French)? If not, how can the analyst specify when and why it applies?

Sapir at one point makes a half-hearted qualification of this idea. Alluding to the fact that in his conceptual translation he includes even such things as gender, he remarks that he has 'exaggerated somewhat'. 'It goes without saying', he adds, 'that a Frenchman has no clear sex notion in his mind when he speaks of un arbre ('a-masculine tree') or of une pomme ('a-feminine apple').' [...] - One is led to wonder, of course, how Sapir managed to distinguish between what is and what is not thus 'exaggerated' in his conceptual translations. (Cook, 1978b, p.34; endnote 38) 
We typically would use the term literal translation if we want to indicate that the English words that are selected occupy a grammatical category and are arranged in a grammatical sequence which is approximately equivalent to the grammatical arrangement of a sentence from another language. In other words, the Yoruba language might accommodate constructions of a kind that do not correspond in any straightforward oneto-one fashion to constructions in English. In this way, these 'literal' translations may perspicuously display some of the differences between the two grammars (construed in terms of ordinary linguistic categories such as ‘noun', ‘adverb’, or 'verb’). For example, in order to display different locations of the verb in English or German sentences we may translate a German utterance not as "I want to know his name”, but 'literally' as "I want his name know".

However, as already pointed out, this kind of grammatical transliteration does not seem to be what Watson has in mind. Watson is less interested in comparing different grammars as such, than in comparing implicit ontologies and her translations seem to be designed to render what Yoruba-speaking says about the nature of the world rather than the specific differences in grammatical structures between the two tongues. Thus, an answer to the question "What is it?” in Yoruba that could (and apparently for all practical purposes would) be translated into English as "It is water", is rendered as:

"Watermatter (matter with the characteristics of waterness) here manifests its inner, intrinsic and permanent nature” (p.289).

Alternatively, "It is a hoe" is rendered as:

"Hoematter (matter with the characteristics of hoeness) here manifests its inner, intrinsic and permanent nature” (p. 289).

Greiffenhagen, C. and W. Sharrock (2007). Linguistic relativism: logic, grammar, and arithmetic in cultural comparison. Language \& Communication 27 (1), 81-107. 
We remain unclear as to how Watson arrives at these 'translations' or 'renderings'. There seem to be two possibilities. Firstly, the Yoruba are actually voicing just these words. On that count, all previous translations from the Yoruba into English (e.g., as simply "It is a hoe") are in a profound sense wrong, and Watson is claiming that she is the first one to provide a correct translation. That seems rather odd.

Alternatively, and secondly, the Yoruba might not be actually saying this, but perhaps Watson wants to say that this is what they mean when they say these words - it is 'encoded' in those words. In that case it seems at least as plausible that it is not the Yoruba that mean this - but it is Watson 'reading into' ordinary Yoruba discourse her own metaphysical assumptions. In our view, it is Watson who insists that this is what the Yoruba must be meaning by what they are saying (even though Yoruba-speakers are not themselves aware that they must be meaning this - see below). We are saying this not on the basis of our knowledge of the Yoruba language, but on the basis of our knowledge of the English language, for which Watson claims the following:

When one is an English-speaker and fingers are held up in code for separated material items, their separateness and spatiotemporal uniqueness is inevitably commented upon by English-speakers in designating. (p. 305)

In our view, an English speaker, who is asked "How much water is coming out of that tap?” and replies "five gallons a minute”, is not making any additional comment of the order "five spatiotemporally segregated, discrete and unique units” (and English speakers are presumably well aware that fingers are subdivisions of the hand). Consequently, Watson's translations are not 'translations' in the traditional sense but are 'metaphysical renderings'. Our problem with Watson has less to do with faithfulness to the form of the 
Yoruba original and more with the English into which the Yoruba is purportedly rendered. No matter what the Yoruba original, we cannot see that Watson's translations provide any intelligible rendering of that into English, since there seems to be no (distinctive) sense to it. In English, does "water 'manifests its inner, intrinsic and permanent nature”" differ significantly (in meaning or substance) from "there is some water”? In English, “its inner, intrinsic and permanent nature” has no genuine semantic value - for what might reference to that 'nature' possibly be? What, in English, could the latter phrase add to "there's some water"? Does "its inner, intrinsic and permanent nature” refer to liquidity, wetness of touch, or colourlessness? However, if so, why call these constituents of the 'inner' nature of water rather than less portentously rendered as standard characteristics of water (the same characteristics for the Yoruba as for us, perhaps)?

\section{Describing the world correctly}

Some of these confusions can be explained through Watson's peculiar picture of language. Above, we had pointed out that for Watson language is a tool to talk 'about' the world. In other words, for Watson, the primary function of words is to identify things. However, taking her intellectualist-metaphysical project one step further, Watson seems to assume that in talking about the world ordinary people are also expressing their preconception of what kinds of things the world is made of. We are saying this, because according to Watson language is not just used in order to talk 'about' the world, but its use presupposes the speakers' conviction that they to do so in a 'correct' way:

An English-speaking person might use natural number in quantification and say 'four oranges are there in the bowl', or 'I would like one

Greiffenhagen, C. and W. Sharrock (2007). Linguistic relativism: logic, grammar, and arithmetic in cultural comparison. Language \& Communication 27 (1), 81-107. 
kilogram of oranges, please', and she would feel confident that she is talking about what there is in the world, in a precisely correct way. [...] When you talk in English, there is a right and a wrong way to talk of things and their qualities, and use numerals in that talk. There is a right and a wrong way, not because the world is organized in a particular way, but because English-speaking people engage in particular organizing practices and say it is organized in that way. (Watson, 1990, pp. 300-301; our emphases) ${ }^{13}$

What is Watson saying here? Is she pointing to the fact that the English speaker is confident that there are four (and not three or five) oranges? Or that the English speaker apart from saying 'how many' oranges there are, has also made a statement about oranges (e.g., that they are 'spatio-temporal particulars')? Has the English speaker also said something about the nature of oranges (e.g., that they possess the quality numerosity)?

We think that the contrast of 'the world being organised in a particular way' and 'saying it is organised that way' is a completely false, wholly contrived, contrast. Of course it is wholly correct to say that there are ten dogs here, because these are dogs and we have correctly enumerated them according to the rules of the natural numbers. However, on what basis could speakers of a language assure themselves that their language speaks of the world in 'precisely the correct' way? How could we (or the Yoruba) tell that our tongue (and its implied ontology) successfully captures the structure of the world? What criteria could be applied to show than the way in which one language identifies basic constituents is evidently preferable to the other? In other words, what could possibly go

\footnotetext{
${ }^{13}$ See also the quotation by Whorf on page 12 above.
} comparison. Language \& Communication 27 (1), 81-107. 
to show that the world really is made up of sortal particulars as opposed to spatiotemporal ones?

These questions are, of course, meaningless. Neither language is true or false. Our confidence in the correctness of what we say in the language derives not from an understanding of the way in which the language divides up the world but from our capacity to speak the language. An English speaker can perhaps be confident that he is talking about the world in a precisely correct way when, like Wittgenstein, they respond to the question “How do you know that is red?” with "I have learned English”. Learning the word 'red' in English involves - amongst many other things - being able correctly to identify samples of red (that colour is called 'red'). More fully, it is called red in English. It is called 'rouge' in French. Perhaps some languages do not have any name for 'red', or perhaps the name they have does not entirely coincide in the way 'red' is distinguished from other colours. Again, the truly surprising thing would be a people who apparently lacked colour vision, who made no colour discriminations, or had nothing that could be called a colour vocabulary - not ones whose colour vocabulary can be co-ordinated with ours.

In what sense could someone who speaks of colours in English go wrong merely by virtue of their use of the colour vocabulary, and in what corresponding way could the user of another colour vocabulary go right (except in respect of the application of the colour vocabulary being used)? There is no place in the discourse for the assertion that this colour 'really is red' rather than 'rouge'. After all, does it even make sense to say that this really is red rather than maroon, or really red rather than scarlet? That distinctions can be made does not mean that they have to be, nor that the divergence between people comparison. Language \& Communication 27 (1), 81-107. 
who use those distinctions and those who do not is one of ontology. Nothing is required beyond competence in the language to give additional confidence that the English colour vocabulary itself 'speaks correctly of the world'. To say this is not to say that something additional has been done to assure that the way the world is corresponds to the classifications of the language, for this could only be an aimless effort when the colour vocabulary defines (so to speak) how the distinctions that one makes 'speak correctly' of one colour or another ('of the world' is just a superfluous characterisation). The fact that some other people do not make the colour distinctions that we do does not detract from or carry any implications for the fact that we make these (after all, most of us do not - could not - routinely make many of the colour discriminations that an English painter or printer needs as a matter of course: see a printer's colour index).

On the other hand, and just to emphasise that we do not suppose that knowing the language is sufficient to license empirical claims, a correct answer to the question "How do you know that table is four feet six inches long?” is not "I speak English”, but "I have measured it". It would, though, be nonsense for the questioner to continue: "Yes, you have measured it, and according to you it is four feet six inches long, but does imperial measurement really describe how the world is? Perhaps the table is made of centimetres and millimetres instead?”.

The most tangibly distinctive feature of Yoruba number-based practice that Watson (1990) reports seem to be in the standardised quantities into which commodities are packaged (e.g., into twenties, rather than say dozens or half dozens). 
A Yoruba fruit-seller will quote prices for oranges in groups of five (osòn márùn) or twenty (ogún osòn), for in Yorubaland that is how oranges are generally sold. (p. 301)

However, no one is going to suggest that oranges come naturally divided into sixes and twelves rather than fives or twenties, or that the difference in these units entails a difference in worldview. Why would someone who sells oranges in half-dozens imagine that they were 'describing the world correctly'? (Even though they would surely have no reason, either, to worry that they were 'describing the world incorrectly'.)

In sum, we find it difficult to discern what Watson could mean by saying that there is "a right and a wrong way to talk of things and their quality”. Watson's whole account wanders unstably between arguing:

(1) By speaking any language one cannot avoid applying an ontology. In other words, we, when using language correctly, are thereby automatically describing the world correctly (according to our supposed implicit ontology). However, if that is the case, what role does the ontology play? Would it not be enough to say that we speak the language (correctly)?

(2) In order to speak, one needs to be aware of what kinds of things one might be talking about. In other words, we, when using language correctly, are simultaneously thinking about what kinds of things there are in the world. However, this would seem to contradict Watson's claim that the ontologies are “implicit” so that “we rarely become aware of them”. 


\section{The indiscernibility of ontologies}

The striking thing about the situation as described by Watson is that the habitation within different ontologies (among the English and Yoruba) seems to be a distinction without a (practical) difference ${ }^{14}$. Strikingly, this difference has previously gone unnoticed, which might seem surprising, given that there has been intense interlingual contact, translation and bilingualism for a long time. Watson herself notes this:

I have described a profound difference between use of the Yoruba language and that of the English language. Why has this basic difference between English and Yoruba not previously been pointed out? There are, after all, several million people who are, to varying degrees, bilingual in English and Yoruba. (Watson, 1990, p. 290; our emphases)

Further

An English-speaking person and a Yoruba-speaking person handle a cup of water in the same way, and they might wield a hoe in a similar fashion. But, despite these similarities of physical manipulation, they talk of them in different ways because, in the practice of using their language to refer, they allocate primacy to different aspects of the matter they say is in the world. (p. 290)

In other words, Watson acknowledges that the 'profound difference' does not show up in the practical lives supposedly separated by it. Watson has a ready, but not convincing, explanation as to why this has gone unnoticed:

\footnotetext{
${ }^{14}$ Again, Watson seems to follow Quine's theoretical scepticism: "stimulus meaning was incapable of deciding among 'rabbit', 'rabbit stage', and various other terms as translations of 'gavagai'. If by analytical hypothesis we take 'are the same' as translation of some construction in the jungle language, we may proceed on that basis to question our informant about sameness of gavagais from occasion to occasion and so conclude that gavagais are rabbits and not stages. But if instead we take 'are stages of the same animal' as translation of that jungle construction, we will conclude from the same subsequent questioning of our informant that gavagais are rabbit stages. Both analytical hypotheses may be presumed possible." (Quine, 1960, pp.71-72)
}

Greiffenhagen, C. and W. Sharrock (2007). Linguistic relativism: logic, grammar, and arithmetic in cultural comparison. Language \& Communication 27 (1), 81-107. 
The explanation lies in the fact that the categorical scheme inherent in language is usually held unreflectively by speakers of that language - it is the taken-for-grantedness implicit in speaking that language. It is not common for speakers of a language to examine what types of material objects their language commits them to. Rather, the difference will be noticed as a difficulty in translation. (Watson, 1990, p.290; our emphases)

Our question is this: How could people genuinely inhabit substantially different worlds and yet this difference not show up in the form of practical difficulties in the transactions between them? If there really were 'fundamental' differences in the underlying ontologies, we would imagine that providing for discrepant ontologies would by now be an integral and well documented feature of innumerable areas of cross-language contact. The practical and empirical intangibility of this supposed ontological gulf between languages has the marks that Wittgenstein thought identified a philosophical nonproblem: such arguments characteristically claim profound and deeply perturbing implications that turn out to disturb nothing and no-one since they lack any factual content.

Watson's explanation that the difference will only be noticed as though it were 'only' one of translation hardly begins to respond to this issue. It might seem minimally plausible that monolingual communities isolated from all other contact might naively suppose that their language's ontology represents the way the world intrinsically 'is'. However, how can they continue in this supposition when they come across people who live in a supposedly discontinuously different world from them? How could they fail to notice that other people - other languages - have discrepant ontologies, and how, therefore, continue to be unaware of what their own ontology is? How could bilinguals avoid noticing the fact that that the character of the natural world is transformed every time 
they switch tongues? Indeed, wouldn't the difference be starkly manifest in the kinds of things their respective remarks actually say?

Apparently, they do notice this difference, though, from Watson's formulation it seems as if they do so only occasionally, even rarely, and even when they do they fail to recognise the true nature of the difference mistaking it (only?) for 'a difficulty in translation'. One would have thought translation would exacerbate the difficulty, not diminish it, for the capacity of one language to capture the sense of a language postulating a discrepant ontology should surely need to be practically provided for. If, as Watson supposes, the speakers of a language suppose that their language describes the world in a 'precisely correct' way, how could those speakers possibly be comfortable with another language which must, by the same token, describe the fundamental nature of the world incorrectly? How can the fact that people are 'worlds apart' fail to register every time there are interlingual events: shouldn't they - somewhere - collide?

The difference is not at the lexical level, for there are Yoruba/English dictionaries which register other differences between the two languages, but omit to mention this one, and proceed to provide straightforward Yoruba-English matches for large parts of the vocabulary: e.g. 'a calabash is a utensil or container made from the dried, hollowed-out shell of any of these fruits' (African Heritage Dictionary), and oko = hoe (Barber, 1984, p.65).

Furthermore, and more importantly, the English and the Yoruba seem to, for all practical purposes, individuate in identical ways. In Watson's own words:

Greiffenhagen, C. and W. Sharrock (2007). Linguistic relativism: logic, grammar, and arithmetic in cultural comparison. Language \& Communication 27 (1), 81-107. 
The use of these [Yoruba] terms as subjects implies that a specific stimulus situation is being talked of. Here spatiotemporal situation becomes a defining characteristic of the sortal particular designated. In this situation, sortal particularity coincides with spatiotemporal particularity, which results in a similarity between ó and won in Yoruba-language talk, and 'it' and 'they' in English-language use. (p. 296; our emphases)

In other words, Yoruba speakers make the same differentiations between ten and nine calabashes of water as the English do. They can tell the difference between a calabash of water and a lake full of water. They can taste the difference between water plain and mixed with orange. They can tell that the stuff pouring off the cliff is the same as that splashing into the bath, and so on.

Equally, the supposedly primary status of the sortal or the spatiotemporal cannot involve any difference in perceptual processing, as though Yoruba actually mentally start from noting the presence of dog stuff and then determine the mode of manifestation (i.e., it is dogstuff subdivided into separate dogforms). It is no wonder when 'noticed' this divergence of ontologies would be taken for 'a difficulty in translation', for the only way in which it could be detected is by some more-than-usual translational incongruity: otherwise there is nothing else in respect of the configuration of the observable scene to discern. The two worlds are observationally equivalent in their individuations and enumerations.

When Watson writes:

In translation $k a ̀$ is usually rendered as 'counting', and wòn as 'measuring'. We can now see that this is misleading, because the categorical scheme which underlies kà and wòn is quite different to that which underlies counting and measuring. However, if one is happy to acknowledge that the basis of translation is physical operation, and not

Greiffenhagen, C. and W. Sharrock (2007). Linguistic relativism: logic, grammar, and arithmetic in cultural comparison. Language \& Communication 27 (1), 81-107. 
be tempted to read more into it than merely that [our emphasis], we can see that these translations are apt. (Watson, 1990, p. 299)

Our answer would be: Why would anyone need to read anything more into it? Who needs to retain a parallel track relation between what we say and what we (must) mean, as though the thought that 'the basis' of translation either is, or is not, manifest in a 'physical operation'.

\section{Conclusion}

Our argument has focussed on testing the sense of Watson's claim that Yoruba speakers possess a different ontology, i.e., that they possess a different conception of what kinds of things make up the substructure of the observable world. The difference postulated by Watson is not at the level of vernacular objects (ordinary things), i.e., the claim is not that the Yoruba have different dogs, oranges, or hoes than the English. Nor is the difference at the level of commonplace substances (what the objects are made up), e.g., a hoe made out of plastic versus a hoe made out of wood. No, the difference is supposed to be on the ontological level and is taken to imply divergences in the understanding (as best we can phrase it) of the 'order of being' of the ultimate constituents of ordinary things.

A crucial sub-text of our argument has been that when it comes to making cross-cultural comparisons social scientists rather tend to neglect the fact that such comparisons are unavoidably two-sided. The urge to destabilise our own supposedly absolutist convictions requires dramatic disparities with the other culture's framework of thought, and this encourages exaggeration, if not distortion, of what can be gleaned from the few arbitrarily selected and thinly described features of the other culture that are made available. Often more important to these contrasts, however, is the way in which our own comparison. Language \& Communication 27 (1), 81-107. 
culture is pictured, with it being assumed that some sketchily set out philosophical positions can adequately stand for how we think. Thus, our argument must not be misunderstood as insisting that the Yoruba can't have a different ontology to us and that, therefore, they must have the same ontology as us. We argue, rather, that the Yoruba have neither the same nor a different ontology to ours because we do not have one: there is no showing of why it is that, when we speak English, our speaking depends upon presupposing, holding or applying this alleged ontology. This same point applies to the Yoruba case. As Cook remarks with respect to Whorf's linguistic relativism:

[Whorf] takes it for granted that Aristotle and other philosophers have correctly discerned and made explicit the metaphysics contained in our Indo-European languages. (If they have gone wrong, it is only when they assume that this is the only possible, and hence the correct, metaphysics). [...] It never occurred to him to suspect that Aristotle and others may have gone wrong in seeing an implicit metaphysics in the grammar of Indo-European languages. (Cook, 1978b, p.6)

The role of ontologies in the respective languages seems akin to that of Wittgenstein's notorious 'beetle in the box' example: it plays no practical part in the casual conversations, commercial transactions, agricultural projects, games or other affairs that involve cross language contact and bilingualism and thus can be cancelled out (it is a difference that makes no difference). This, though, points to the deeper problem Wittgenstein's philosophy would pose for this philosophical project of linguistic relativism: to show that any language can intelligibly be said to make 'reference to the ultimate nature of things'. Dilman (2004) nicely summarises the reason why empiricist philosophers misread the structure of natural language as they do, at the same time highlighting the strangeness of Watson's conception of Yoruba:

Greiffenhagen, C. and W. Sharrock (2007). Linguistic relativism: logic, grammar, and arithmetic in cultural comparison. Language \& Communication 27 (1), 81-107. 
The empiricist philosopher goes beyond the bounds of nonphilosophical empiricism in seeking [...] an ultimate justification of what it must be possible to attain empirically. He mislocates the concept of physical reality - a formal concept - by treating it as an ordinary empirical concept, signifying a class of objects such as trees, mountains, houses etc. (p. 172)

As Michael Williams (2004) further explains:

[...] 'physical object' is not the concept of a kind of object, like 'unicorn' or 'planet'. 'Physical object' is a piece of logical or semantic vocabulary, thus unsuitable for formulating the empirical hypothesis the sceptic or idealist would like to express. (p. 86)

In other words, the expression 'object' is parasitic on expressions like 'chair', 'orange', 'bucket' and is not one which must be acquired prior to their use (not least since the meaning of 'object' is explained by reference to chairs, tables, books, etc.). Watson's account of language and, especially, of language acquisition owes much to the wholly philosophical picture painted in Quine’s (1960) empiricism.

The idea that speakers of a language must posit an ontology (a metaphysical theory) in order to understand what they are speaking about when they make reference is also not worked out so far as to explain how it is that learners of a language manage to hit on the same ontology for a common language. That is to say, how is a child who is acquiring language to determine what kind of ontology underpins the referential expressions of a language? After all, it is necessary for Watson, through analysing the work of predicators in English-language usage and Yoruba-language usage, to "infer the extension of designating terms used in utterances” (p. 289). So supposedly would learners of either language. However, what would ensure correct inference here, especially when, as Watson observes: 
People walk around and sit upon chairs, and put their arms around people irrespective of what they may say is in the world. Possibly too they have similar pictorial images in their minds An English-speaking person and a Yoruba-speaking person handle a cup of water in the same way, and they might wield a hoe in a similar fashion. But despite these similarities of physical manipulation, they talk of them in different ways because, in the practice of using their language to refer, they allocate primacy to different aspects of the matter they say is in the world. (Watson, 1990, p. 290; our emphases)

Watson's argument is circular: the child learning language must postulate an ontology in terms of which to construe the expressions in the language, but, if ontology varies with language, then the child must extrapolate the correct ontology from the grammatical structure of that language. In short, there is no real difference between showing that two communities of language users speak of different things and showing that they speak of the same things in different ways.

In sum from Watson's account we learn remarkably little about the Yoruba (i.e., their culture and associated practices) - except that their language seems to have a different grammar than ours. Instead, we learn a lot about philosophical assumptions (confusions) of the analyst.

\section{Acknowledgements}

Wes Sharrock would like to thank the Arts and Humanities Research Board (AHRB) for an Innovation Award (grant number B/IA/AN10985/APN17690) to support the work reported in this paper.

\section{References}

Barber, K. (1984). A Beginners Course in Yoruba. New Haven: Yale University Press. Bloor, D. (1976). Knowledge and Social Imagery. London: Routledge.

Greiffenhagen, C. and W. Sharrock (2007). Linguistic relativism: logic, grammar, and arithmetic in cultural comparison. Language \& Communication 27 (1), 81-107. 
Cook, J. W. (1978a). Whorf's linguistic relativism. Philosophical Investigations 1 (1), 1-30.

Cook, J. W. (1978b). Whorf's linguistic relativism: Part 2. Philosophical Investigations 1 (2), $1-$ 37.

Dilman, I. (2004). Wittgenstein and the question of linguistic idealism. In D. McManus (Ed.), Wittgenstein and Scepticsm, pp. 162-177. London: Routledge.

Graves, R. (1960). Comments on "Lineal and nonlineal codifications of reality". In E. Carpenter and M. McLuhan (Eds.), Explorations in Communication: An Anthology, pp. 155-161. Boston: Beacon Press.

Greiffenhagen, C. and W. Sharrock (2006). Mathematical relativism. Journal for the Theory of Social Behaviour 36 (2), 97-117.

Greiffenhagen, C. and W. Sharrock (forthcoming). Logical relativism.

Joseph, J. E. (2002). The sources of the 'Sapir-Whorf hypothesis'. In From Whitney to Chomsky: Essays in the History of American Linguistics, pp. 71-105. Amsterdam: John Benjamins.

Lee, D. (1960). Lineal and nonlineal codifications of reality. In E. Carpenter and M. McLuhan (Eds.), Explorations in Communication: An Anthology, pp. 136-154. Boston: Beacon Press.

Lenneberg, E. (1953). Cognition in ethnolinguistics. Language 29 (2): 463-471.

Macbeth, D. H. (2002). A commentary on instructional design. Journal of the Learning Sciences 11 (2\&3): 373-386.

Quine, W. (1960). Word and Object. Cambridge, MA: MIT Press.

Reed, H. J. and J. Lave (1979). Arithmetic as a tool for investigating relations between culture and cognition. American Ethnologist 6 (3): 568-582.

Sharrock, W. W. and D. C. Anderson (1982). The persistent evasion of technical issues in media studies. Sociology 16 (1): 108-115.

Verran, H. (2000a). Logics and mathematics: challenges arising in working across cultures. In $\mathrm{H}$. Selin (Ed.), Mathematics Across Cultures: The History of non-Western Mathematics, pp. 5578. Dordrecht: Kluwer.

Verran, H. (2000b). Accounting mathematics in West Africa: some stories of Yoruba number. In H. Selin (Ed.), Mathematics Across Cultures: The History of non-Western Mathematics, pp. 345-371. Dordrecht: Kluwer.

Watson, H. (1987). Learning to apply numbers to nature: a comparison of English speaking and Yoruba speaking children learning to quantify. Educational Studies in Mathematics 18 (4): 339-357.

Watson, H. (1990). Investigating the social foundations of mathematics: Natural number in culturally diverse forms of life. Social Studies of Science 20 (2): 283-312.

Whorf, B. L. (1956). Language, Thought and Reality: Selected Writings of Benjamin Lee Whorf (Edited by B. J. Carroll). Cambridge, MA: MIT Press.

Williams, M. (2004). Wittgenstein's refutation of idealism. In D. McManus (Ed.), Wittgenstein and Scepticism, pp. 76-96. London, Routledge.

Wittgenstein, L. (1953). Philosophical Investigations (Translated by G.E.M. Anscombe). Oxford: Basil Blackwell. 